\title{
Pesquisas sôbre a análise estatística. de experiências de adubação com o auxílio da Lei de Mitscherlich
}

\author{
FREDERICO PIMENTEL GÖMES \\ Cadeira de Matemática
}

e

EURIPEDES MALAVOLTA

Seç̧ăo Técnica de Química Agrícola da Escola Superior de Agricultura "Luiz de Queiroz" Universidade de São Paulo

INDICE

1 -Introdução ....................... 2

2-As experiências analisadas $\ldots \ldots \ldots \ldots \ldots, 2$

3-Crítica dos resultados obtidos ........... 10

4-Apêndice $. . \ldots \ldots \ldots \ldots \ldots \ldots \ldots \ldots \ldots, 11$

5 -Abstract ........................ 12

6 -Bibliografia citada $\ldots \ldots \ldots \ldots \ldots \ldots \ldots, 13$ 


\section{1 - INTRODUÇÃo}

Este estudo resultou de uma sugestão do Prof. Luiz de Freitas Bueno, apresentada no "Terceiro Seminário de Estatística" por ocasião da discussão de um trabalho de Pimentel Gomes (1950). Os dados analisados foram gentilmente cedidos pelo Dr. Raul Edgard Kalckmann, do Ministério de Agricultura, e na análise estatística contamos com a colaboração amiga do Prof. F. G. Brieger e do Engenheiro-Agrônomo Izaias Rangel Nogueira.

O fim principal visado foi verificar se é justificada a hipótese de que os valores obtidos para a variância em cada nível de uma experiência de adubação com diferentes doses de um mesmo adubo podem ser tomadas como estimativas de u'a mesma variância, comum a todo o ensaio. Durante a pesquisa, porém, surgiram outros itens interessantes, que exigiram a cooperação de Euripedes Malávolta.

Os métodos seguidos são os indicados em trabalhos anteriores (Pimentel Gomes e Malavolta, 1949 a e 1949 b).

\section{2 - AS EXPERIENCIAS ANALISADAS}

Estudamos uma experiência de trigo realizada em Ponta Grossa, Paraná, pelo Ministério de Agricultura, na qual se aplicou cal extinta nas doses de 0, 2, 4, 6 e 8 toneladas por hectare. Utilizou-se um quadro latino de $5 \times 5$. A cal foi aplicada em julho de 1940 e o trigo foi cultiyado de 1940 a 1948 sempre nas mesmas parcelas. De 1940 a 1945 semeou-se a variedade Fronteira, e de 1946 a 1948 foi cultivada variedade Trintecinco. Perdeu-se a cultura em 1946.

Anualmente se fazia uma adubação geral nas doses seguintes :

Salitre do Chile
Superfosfato
Sulfato de potássio
$100 \mathrm{~kg} / \mathrm{ha}$,
$350 \mathrm{~kg} / \mathrm{ha}$,
$80 \mathrm{~kg} / \mathrm{ha}$

As médias obtidas em 8 anos de ensaios foram as que constam do quadro seguinte, onde estão expressas em quilos por hectare. 


\begin{tabular}{l|r|r|r|r|r}
\hline & & \multicolumn{5}{|c}{ Doses de cal } \\
\cline { 2 - 6 } Anos & 0 & & & & \\
\hline & & & & & \\
1940 & 103 & 118 & 1.06 & 104 & 8 \\
1941 & 513 & 745 & 788 & 726 & 112 \\
1942 & 984 & 1.386 & 1.458 & 1.486 & 1.464 \\
1943 & 886 & 1.093 & 1.273 & 1.313 & 1.378 \\
1944 & 356 & 640 & 604 & 672 & 690 \\
1945 & 596 & 744 & 830 & 844 & 814 \\
1947 & 2.040 & 2.378 & 2.658 & 2.724 & 2.798 \\
1948 & 1.480 & 1.650 & 1.630 & 1.780 & 1.790 \\
\hline
\end{tabular}

Em 1940 a influência da calagem fol absolutamente nula, naturalmente por ter sido feita com antecedência insuficiente. Já em 1941 se nota aumento na produção das parcelas adubadas, mas aparentemente houve efeito tóxico do adubo nas doses mais elevadas. Utilizando-se só as três primeiras doses para o cálculo, a equação de Mitscherlich obtida foi

$$
y=798,4\left[1-10^{-0,36365(x+1,2302)}\right]
$$

O cálculo feito com as cinco doses deu, porém

$$
y=756,1\left[1-10^{-1,60906(x+0,2920)}\right]
$$

O teste de Bartlett, nos moldes indicados por Conagin (1949), foi feito a seguir, obtendo-se os valores abaixo discriminados.

\begin{tabular}{c|c|c}
\hline Tratamento & Variáncia & log \\
& & variância \\
\hline 0 & $21.053,2$ & 4,3234 \\
2 & $3.142,0$ & 3,4972 \\
4 & $21.733,2$ & 4,3371 \\
6 & $20.314,8$ & 4,3078 \\
8 & $12.014,8$ & 4,0797 \\
\hline Total & $78.258,0$ & 20,5452 \\
\hline
\end{tabular}


Temos entáo

$$
\begin{aligned}
& \overline{\mathrm{s}}^{2}=\frac{78,258}{5}=15,652, \\
& \log 15,652=4,1946, \\
& \mathrm{~d}=2,303 \times 4[5 \times 4,1946-20,5452] \\
& =3,94, \\
& \quad \mathrm{C}=1,1 .
\end{aligned}
$$

Logo, temos

$$
\chi^{2}=\frac{3,94}{1,1}=3,58, \mathrm{~g}=4
$$

\begin{tabular}{|c|c|c|c|}
\hline $\begin{array}{l}\text { Causa de } \\
\text { variação }\end{array}$ & $\begin{array}{l}\text { Grau de } \\
\text { liberdade }\end{array}$ & $\begin{array}{l}\text { Soma dos } \\
\text { quadrados }\end{array}$ & $\begin{array}{l}\text { Quadrado } \\
\text { médio }\end{array}$ \\
\hline $\begin{array}{l}\text { Linhas } \\
\text { Colunas } \\
\text { Tratamentos } \\
\text { Residuo }\end{array}$ & $\begin{array}{r}4 \\
4 \\
4 \\
12\end{array}$ & $\begin{array}{r}10.242,6 \\
20.982,2 \\
236.522,2 \\
47.033,2\end{array}$ & $\begin{array}{r}2.561 \\
5.246 \\
59.131 \\
3.919\end{array}$ \\
\hline Total & 24 & $314.780,2$ & \\
\hline
\end{tabular}

O limite de $5 \%$ é 7,8 e o de $1 \%$ é 13,3. Logo, o valor obtido é insignificante, o que evidencia 0 fato de que podemos aceitar a hipótese de que os tratamentos não influiram sôbre a variancia. Podemos então fazer uma análise de variância segundo os métodos correntes, como a seguinte.

Naturalmente há necessidade de decompor, a seguir, a variancia atribuida aos tratamentos, isolando a parte devida à correlação, de acôrdo com o que demonstrou Pimentel Gomes (1950). Obtemos, então; os quadros seguintes. 


\begin{tabular}{l|c|c|c|c|c}
\hline & 0 & 2 & 4 & 6 & 8 \\
\hline Valores esperados & 499,8 & 749,8 & 755,9 & 756,1 & 756,1 \\
\hline Mèdias cbservadas & 513,4 & 745,0 & 788,4 & 725.8 & 745,2 \\
\hline
\end{tabular}

\begin{tabular}{c|c|c|c}
\hline $\begin{array}{c}\text { Causa de } \\
\text { variação }\end{array}$ & $\begin{array}{c}\text { Grau de } \\
\text { liberdade }\end{array}$ & $\begin{array}{c}\text { Soma dos } \\
\text { quadrados }\end{array}$ & $\begin{array}{c}\text { Quadrado } \\
\text { médio }\end{array}$ \\
\hline $\begin{array}{c}\text { Regressão pela } \\
\text { lei de } \\
\text { Mitscherlich }\end{array}$ & 2 & $225.030,8$ & $112.515^{* *}$ \\
\hline $\begin{array}{c}\text { Desvios a partír } \\
\text { da curva de } \\
\text { regressão }\end{array}$ & 2 & $11.419,4$ & 5.746 \\
\hline
\end{tabular}

Os desvios a partir da curva de regressão dão uma nova estimativa para a variancia residual, estimativa esta que não deve diferir estatisticamente da estimativa obtida antes pelos métodos correntes. De fato a nova estimativa (5.746) não difere estatisticamente da outra (3.919). Verifica-se, assim, que a lei de Mitscherlich se adapta bem aos dados em estudo e que a adubação produziu um efeito estatisticamente significativo.

Podemos, então, calcular a dose mais econômica de adubo com o auxílio da fórmula

$$
x=\frac{1}{c} \log \frac{A, s c}{f t \log e}-b,
$$

cuja dedução já foi exposta por Pimentel Gomes e Malavolta (1949 a). Nessa fórmula, A, b, c são parâmetros da equação de Mitscherlich, s será, para nós, o preço de um quilo de trigo em grão, t o custo de uma tonelada de cal extinta e f um fator maior que um, que admitimas igual a 1,5. Apenas para exemplificação, tomamos $t=300$ (cruzeiros por tonelada de cal extinta) e $s=2,50$ (cruzeiros por quilo de trigo). Obtivemos então 


$$
\mathbf{x 1}=0,449 \text { tonelada },
$$

isto é, a adubação ideal seria de cêrca de 450 quilos de cal por hectare. Nesse cálculo se exige, implicitamente, que o adubo seja pago no primeiro ano, dievendo a calagem, evidentemente, ser feita com a antecedência necessária. Como o efeito da calagem dura muitos anos, êsse cálculo é, talvez, um tanto pessimista. Mas o nosso pessimismo se justifica, até certo ponto, pela escassez de crédito, especialmente a longo prazo, para os nossos lavradores, que necessitam, pois, de pronto reembolso do capital iempregado. A produção esperada com $x=0,449$ é de 707 quilos por hectare. Se nos lembrarmos, porém, de que a adubação feita com superfosfato em 1940 e 1941 corresponde a um teor de, aproximadamente, 270 quilos de $\mathrm{Ca}(\mathrm{OH}) 2$ por hectare, chegaremos à conclusão de que, no caso de não se usar o superfosfato, a calagem deverá ser feita à base de uns 720 quilos por hectare.

Se admitirmos, porém o reembolso da adubação em dois anos, deveremos reduzir $\mathbf{t}$ a metade e obteremos então

$$
\mathbf{x} 2=0,636 \text { tonelada },
$$

isto é, dever-se-iam usar uns 640 quilos de cal extinta por hectare, ou 910, no caso de faltar o superfosfato.

Se adotássemos a lequação calculada só com as três doses mais baixas, isto é, se admitíssemos que houve efeito tóxico para as doses mais elevadas, obteríamos

$$
\begin{aligned}
& \mathrm{x} 1=0,337 \text { tonelada }, \\
& \mathbf{x} 2=1,165 \text { tonelada } .
\end{aligned}
$$

Isso, porém, não parece indicado, poiss a análise estatística demonstrou uma excelente concordância entre os dados obtidos e a curva calculada. $\mathrm{E}$ o uso de todos os dados nos dá um resultado com base experimental mais sólida. Nota-se, porém, que, de qualquer forma, os novos valores de $x 1$ e $\mathbf{x 2}$ não são do ponto de vista prático, muito diferentes dos anteriormente obtidos.

Nos demais anos analisados, a marcha seguida foi inteiramente idêntica e os resultados conseguidos são dados a se guir. 
1942

$$
y=1476,2\left[1-10^{-0,37056(x+1,2836)}\right]
$$

O teste de Bartlett deu

$$
\chi^{2}=4,46-\text { insignificante. }
$$

A análise da variância está resumida no quadro seguinte.

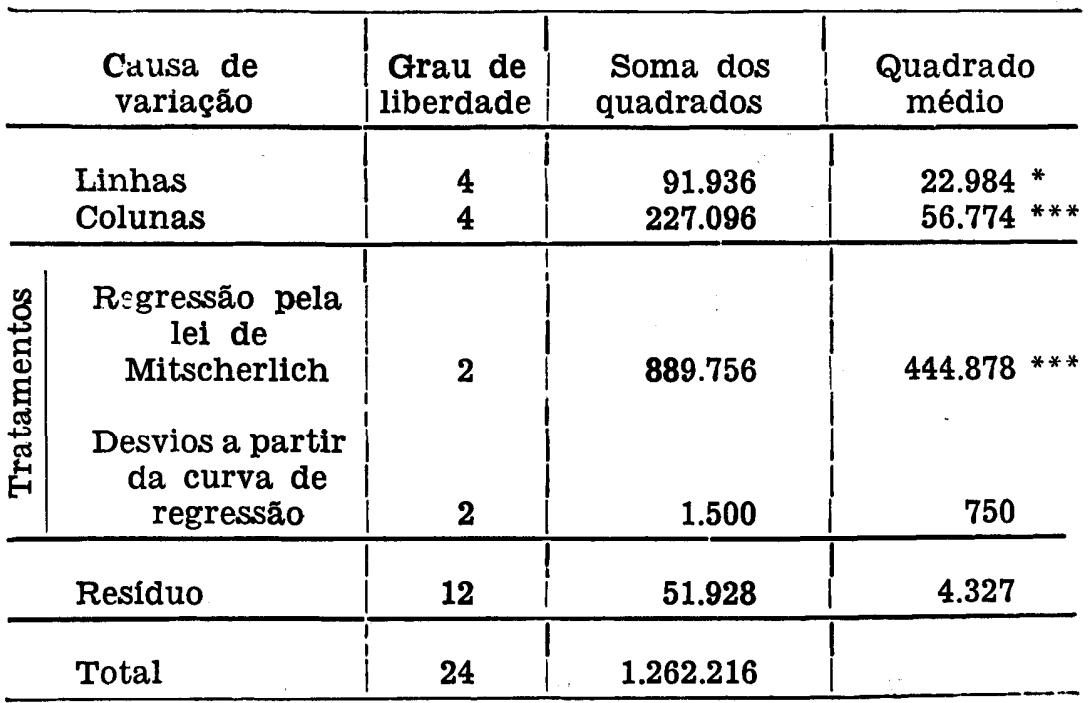

A ađubação mais econômica é

$$
\mathrm{x} 1=0,996 \text { tonelada, }
$$

quando o adubo é pago em um ano e

$\mathbf{x 2}=1,808$ tonelada,

quando pago em dois anos.

1943

$$
y=1442,7\left[1-10^{-0,11230(x+3,6886)}\right]
$$

O teste de Bartlett deu

$$
\chi^{2}=3,83-\text { insignificante. }
$$




\begin{tabular}{|c|c|c|c|c|}
\hline & $\begin{array}{l}\text { Causa de } \\
\text { variação }\end{array}$ & $\begin{array}{c}\text { Grau de } \\
\text { liberdade }\end{array}$ & $\begin{array}{l}\text { Soma dos } \\
\text { guadrados }\end{array}$ & $\begin{array}{l}\text { Quadrado } \\
\text { médio }\end{array}$ \\
\hline & $\begin{array}{l}\text { Linhas } \\
\text { Colunas }\end{array}$ & $\begin{array}{l}4 \\
4\end{array}$ & $\begin{array}{l}180.433 \\
298.355\end{array}$ & $\begin{array}{l}45.108 * \\
74.589 * *\end{array}$ \\
\hline 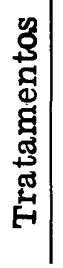 & $\begin{array}{l}\text { Regressão pela } \\
\text { lei de } \\
\text { Mitscherlich } \\
\text { Desvios a partir } \\
\text { da curva de } \\
\text { regressão }\end{array}$ & 2 & $\begin{array}{r}786.704 \\
8.915\end{array}$ & $\begin{array}{c}393.352 * * * \\
4.458\end{array}$ \\
\hline & Resíduo & 12 & 118.540 & 9.878 \\
\hline & Total & 24 & 1.392 .947 & \\
\hline
\end{tabular}

$$
\mathrm{x} 1=0,871 ; \mathrm{x} 2=1,810
$$

1947

$$
\mathbf{y}=2887,3\left[1-10^{-0,12536(x+4,218)}\right]
$$

O teste de Bartlett deu

$$
\chi^{2}=10,54 \text {, duvidoso. }
$$

As variâncias obtidas são dadas a seguir.

Tratamento

0
3
4
6
8
Variancia

63.400

22.370

9.520

16.330

1.470 


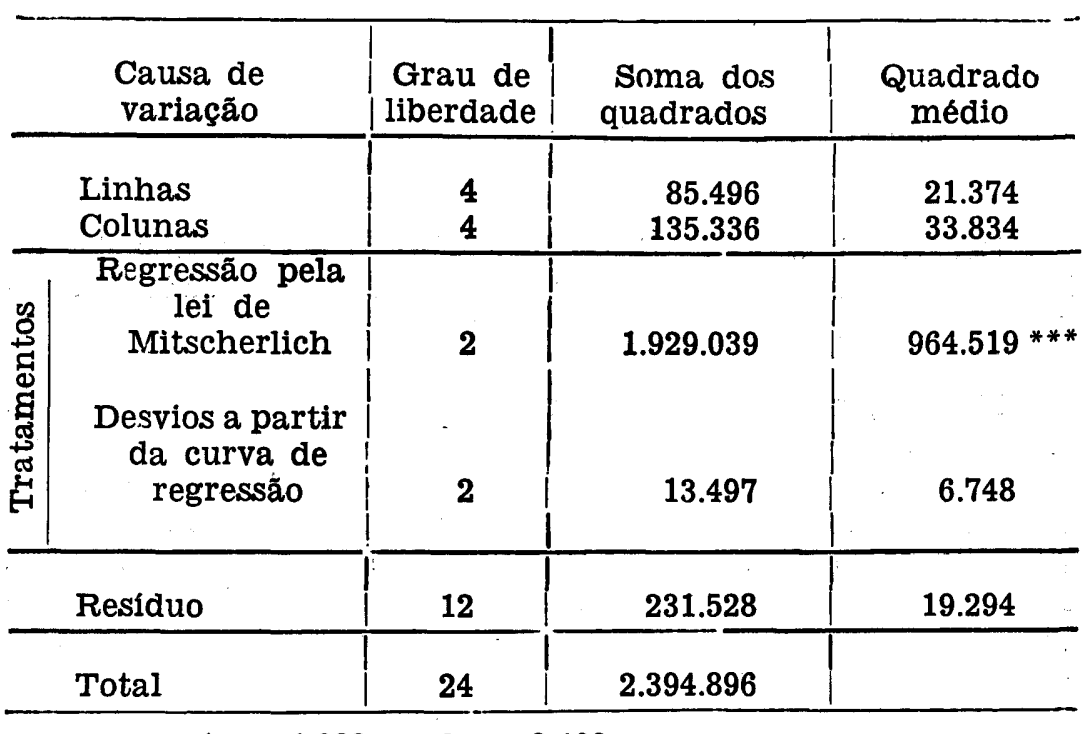

$$
\mathrm{x} 1=1,092 ; \mathrm{x} 2=3,493 \text {. }
$$

1948

$$
y=1909,6\left[1-10^{-0,065968(x+9,4664)}\right]
$$

O teste de Bartlett deu

\begin{tabular}{|c|c|c|c|c|}
\hline \multicolumn{2}{|c|}{$\begin{array}{l}\text { Causa de } \\
\text { variação } \\
\end{array}$} & $\begin{array}{c}\text { Grau de } \\
\text { liberdade }\end{array}$ & $\begin{array}{l}\text { Soma dos } \\
\text { quadrados }\end{array}$ & $\begin{array}{l}\text { Quadrado } \\
\text { médio }\end{array}$ \\
\hline & $\begin{array}{l}\text { Linhas } \\
\text { Colunas }\end{array}$ & $\begin{array}{l}4 \\
4\end{array}$ & $\begin{array}{l}58.600 \\
76.600\end{array}$ & $\begin{array}{l}14.650 \\
19.150\end{array}$ \\
\hline 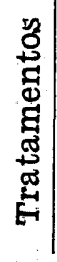 & $\begin{array}{l}\text { Regresssáo pela } \\
\text { lei de } \\
\text { Mitscherlịch } \\
\text { Desvios a partir } \\
\text { da curva de } \\
\text { regressão }\end{array}$ & 2 & 32.623 & $145.675 * * *$ \\
\hline & Residuo & 12 & 95.800 & 7.983 \\
\hline & Total & 24 & 554.973 & \\
\hline
\end{tabular}

$\chi^{2}=1,44$, insignificante. 


\section{3 - CRfTICA DOS RESULTADOS OBTIDOS}

Das análises estatísticas efetuadas, resulta que a lei de Mitscherlich se mostrou sempre aplicável com precisão em todos os anos analisados.

Nota-se, porém, que os parâmetros $\mathbf{A}, \mathbf{b}$, c variam muito. A variação de $\mathbf{A}$ se prende às condições meteorológicas mals ou menos favoráveis nos diversos anos e também à mudança de variedade. $O$ valor de $b$, que mede 0 teor em $\mathrm{Ca}(\mathrm{OH})^{2}$ na testemunha, cresceu invariàvelmente de um ano para outro. asse crescimento se explica, em parte, pelo teor em $\mathrm{CaO}$ anualmente incorporado sob a forma de superfosfato e equivalente, aproximadamente, a 135 quilos, de $\mathrm{Ca}(\mathrm{OH})^{2}$ por hectare. Se descontarmos do valor di $\mathbf{b}$ em cada ano a quantidade de $\mathrm{Ca}(\mathrm{OH})^{2}$ equivalente ao superfosfato já aplicado, obtemos os números que se seguem.

$\begin{array}{ccc}\text { Anos } & \text { Valor de } \mathbf{b} \text { achado } & \text { Valor de } \mathbf{b} \text { corrigido } \\ 1941 & 0,292 & 0,022 \\ 1942 & 1,284 & 0,879 \\ 1943 & 3,688 & 3,148 \\ 1947 & 4,218 & 3,138 \\ 1948 & 9,466 & 8,251\end{array}$

Ainda assim persiste o aumento de $b$, e êsse aumento se explica, na nossa opinião, pela migração do cálcio das parcelas mais adubadas para as menos adubadas e para a testemunha. Sabe-se, aliás, que os complexos humico e alumino-sillcico responsáveis pela acidez e pelo poder absorvente do solo (Page, 1926) se apoderam do cálcio. Este, porém, não permanece parado na terra : move-se tanto verticalmente (Blair e Prince, 1934) como horizontalmente (Miller ie Turk, 1943, pág. 143). De sorte que a migração do cálcio de uma parcela para outra numa experiência, como essa, de parcelas de apenas $100 \mathrm{~m}^{2}$, no campo e durante tantos anos seguidos, é, náo só razoável, camo previsivel. Como consequéncia dessa migraçăo, 
os teores de adubo nas parcelas foram alterados, de sorte que os novos parâmetros apresentam distorsões inevitáveis. Além do b, o parâmetro c foi também atingido, pois decresce extraordinàriamente de um ano para outro.

Em consequência do que acabava de ser exposto, o cálculo da adubação mais econômica só merece fé nos primeiros anos da experiência e especialmente em 1941. Pelos dados obtidos nesse e no ano seguinte vê-se que, no caso do reembolso da adubação em um ano, a calagem deve ser de 500 a 1.000 quilos por hectare. Caso se admite o reembolso em dois anos, pode-se ir até 1.500 quilos ou pouco mais. Não sendo feita adubação com superfosfato, essas doses devem ser aumentadas de uns 300 quilos.

Quanto ao teste Bartlett, os resultados obtidos foram insignificantes em todos as anos, exceto em 1947, quando se obteve um valor de significação duvidosa. Como, porém, os resultados dêsse ano estão, provàvelmente, influénciados pela migração do cálcio, parece-nos razoável aceitar como homogêneas as variâncias nas experiências de calagem de trigo.

\section{4 - APENDICE}

E' evidiente que não havia necessidade de analisar todos os dados. Os dados de 1947 e 1948, especialmente, já se deveriam esperar como pouco representativos do efeito da calagem, devido à provável migração do cálcio de uma parcela para outra e ainda devido à dose de superfosfato, riquíssimo em cálcio, anualmente aplicado. A análise foi feita principalmente com a intenção de examinar como reagiriam os parâmetros da equação num caso como êsse.

Com base em conceitos tradicionais de dinâmica do cálcio no solo, poderia surgir uma objeção visando atribuir os acréscimos da produção, não ao cálcio diretamente, mas às bases que êle deslocaria do complexo coloidal. Se tal se desse, b e c, no caso presente, não teriam significado definido. Entretanto, isso não ocorre. De fato, o cálcio pode fixar-se no solo segundo as reações : 
a) Com troca de iônios $\mathrm{H}$ :

$$
2 \mathrm{H}(\mathrm{Solo})+\mathrm{Ca}(\mathrm{OH}) 2=2(\mathrm{Solo}) \mathrm{Ca}+2 \mathrm{H} 2 \mathrm{O} ;
$$

b) Com troca de iônios metálicos :

$$
2 \mathrm{~K}(\mathrm{Solo})+\mathrm{Ca}(\mathrm{OH}) 2=2 \text { (Solo) } \mathrm{Ca}+2 \mathrm{KOH} .
$$

No primeiro caso, resta sòmente água na fase líquida como produto da reação. No segundo caso, dá-se simplesmente a substituição de uma base por outra. Pràticamente, só a reação (4) se pode produzir nos solos, pois havendo formação de $\mathrm{KOH}$, ou de outro hidróxido qualquer, segue-se logo a condição $\mathrm{K}$ (Solo) por uma reação análoga a (4). Isto foi confirmado experimentalmente por estudos lisimétricos (Demolon, 1948, p. 236; Mc Intyre, 1923).

\section{5 - ABSTRACT}

Statistical analyses of an experiment on wheat were carried out with the aid of Mitscherlich's law. The experiment was made in Ponta Grossa, Paraná, by the Ministry of Agriculture of Brasil. Lime, in the form of $\mathrm{Ca}(\mathrm{OH}) 2$, was applied at the levels of $0,2,4,6$ and 8 metric tons per hectare. A $5 \times 5$ Latin square was used. Lime was applied in 1940 and wheat was cultivated in the same plots for several years. The following fertilizers were annually used for all plots :

\section{NaNO3 \\ Superphosphate K2SO4}

$$
\begin{aligned}
& 100 \text { kilograms per hectare, } \\
& 350 \text { kilograms per hectare, } \\
& 80 \text { kilograms per hectare. }
\end{aligned}
$$

The statistical analysis of the data collected in 1941, 1942, 1943, 1947 and 1948, carried out in accordance with the methods previously introduced by Pimentel Gomes and Malavolta (1949 a, 1949 b) and Pimentel Gomes (1950), proved:

I. That Mitscherlich's law could be correctly applied to the data. 
II. That there was a statistically significant effect of lime on wheat yield.

III. That the optimum amount of lime to be applied to the soil lies between 5 and 15 hundred kilograms of $\mathrm{Ca}(\mathrm{OH}) 2$ per hectare.

IV. That there is a migration of calcium from some plots to others, in such a way that the data obtained in 1947 and 1948 are not representative of the amounts of lime applied in 1940.

V. That the analysis of variance can be used, as the Bartlett test shows that the variances at the distinct levele of lime application are not statistically different.

It must be noted that, with improved variety and fertilization, the yield was rised to about 2500 kilograms per hectare in 1947 , and 1600 in 1948 , being only of about 100 kilograms per hectare in 1940 .

\section{6 - BIBLIOGRAFIA CITADA}

1 - BLAIR, A. W. e A. L. PRINCE (1934). "The influence of lime on the reaction of subsoils". J. Agr. Res. 48, pp. 469-473.

2 - CONAGIN, A. (1949). Teste de Bartlett. Seminários de Estatística Aplicada (1.a série), pp. 75-86.

3-DEMOLON, ALBERT (1948). "Dynamique du soil", $4 a$. edição, Dunod, Paris,

4-MC INTYRE (1923). Soil Sci 16, p. 217, A. Demolon, op. cit., p. 236.

5-MILLAR, C. ERNEST e LLOYD M. TURK (1943). "Fundamentals of Soll Science". John Wiley and Sons Inc., Nova York.

6-PAGE, H. J. (1926). "The nature of soil acidity". Trans. Sec. Comm. Int. Soc. Soil Sci., vol. A, pp. 232-244, Growinger (Holanda). 
7-PIMENTEL GOMES, FREDERICO e EURIPEDES MALAVOLTA (1949a). "Considerações Matemáticas sôbre a Lei de Mitscherlich". Boletim n. 3 da E. S. A. "Luiz de Queiroz". Piracicaba.

8 - PIMENTEL GOMES, FREDERICO e EURIPEDES MALAVOLTA (1949b). "Aspectos Matemáticos e Estatísticos da Lei de Mitscherlich". Anais da. E. S. A. "Luiz de Queiroz", 6, pp. 193-229. Piracicaba.

9 - PIMENTEL GOMES, FREDERICO (1950). "A Lei de Mitscherlich e a Análise da Variância e Experiência de Adubação". Anais da E. S. A. "Luiz de Queiroz", y (a sair).

\section{OBSERVAÇÃO}

Neste trabalho indicamos por um asterisco a significação para o limite de $5 \%$, por dois asteriscos, a significação para o limite de $1 \%$ e por três asteriscos, no caso de $0,1 \%$. 